\title{
ON THE SMALLNESS CONDITION IN LINEAR INVISCID DAMPING: MONOTONICITY AND RESONANCE CHAINS
}

\author{
YU DENG AND CHRISTIAN ZILLINGER
}

\begin{abstract}
We consider the effects of mixing by smooth bilipschitz shear flows in the linearized Euler equations on $\mathbb{T}_{L} \times \mathbb{R}$. Here, we construct a model which is closely related to a small high frequency perturbation around Couette flow, which exhibits linear inviscid damping for $L$ sufficiently small, but for which damping fails if $L$ is large. In particular, similar to the instability results for convex profiles for a shear flow being bilipschitz is not sufficient for linear inviscid damping to hold. Instead of an eigenvalue-based argument the underlying mechanism here is shown to be based on a new cascade of resonances moving to higher and higher frequencies in $y$, which is distinct from the echo chain mechanism in the nonlinear problem.
\end{abstract}

\section{INTRODUCTION}

We are interested in the long-time asymptotic behavior of the linearized 2D Euler equations near monotone shear flows $v=(U(y), 0)$ in a periodic channel $\mathbb{T}_{L} \times \mathbb{R}$ or circular flows $v=r U(r) e_{\theta}$ on $\mathbb{R}^{2}$. After possibly relabeling the log-polar coordinates and considering weighted spaces (see [CZZ19], [Zi117]) both settings can be considered in the framework

$$
\partial_{t} \omega+U(y) \partial_{x} \omega-\beta(y) \partial_{x} \Delta^{-1} \omega=0,
$$

where $\beta(y)=U^{\prime \prime}(y)$ in the plane channel setting. The question of stability of shear flows has a long history, where we in particular mention the stability results of Rayleigh and Fjortoft [Dra02] (for the linearized equations) and of Arnold [Arn66]. Unlike the setting of monotone flows considered in this article, these stability results rely on convexity. More precisely, Rayleigh's and Fjortoft's theorems show that if $U$ is convex, then the linearized Euler equations cannot be exponentially unstable in the sense that there can't exist solutions of (1) of the form $e^{\lambda t} \omega_{0}(x, y)$ with $\Re(\lambda)>0$. However, the solutions may nevertheless be algebraically unstable in the sense $\|\omega(t)\|_{H^{1}} \rightarrow \infty$ as $t \rightarrow \infty$ with a power law rate. Indeed, one explicit example is given by $U(y)=y$.

The stability mechanism which is the focus of this paper instead exploits monotonicity and mixing by shearing. The prototypical example here is given by (TaylorCouette flow, which we recall in the following. In the (Taylor-)Couette flow case $U(y)=y$ and $\beta(y) \equiv 0$ and hence the equation has an explicit solution

$$
\omega(t, x, y)=\omega(0, x-t U(y), y) .
$$

We observe that the $H^{1}$ norm of this solution diverges to infinity as $t \rightarrow \infty$ (for $\partial_{x} \omega(0)$ non-trivial) and the $L^{2}$ norm of $\omega(t)$ remains constant and in particular bounded. However, $\omega(t)$ converges weakly in $L^{2}$ (but not strongly) to its $x$-average. As a consequence due to compactness in the Biot-Savart law the velocity field 
strongly converges to an asymptotic profile as $t \rightarrow \infty$. This phenomenon is known as (linear) inviscid damping. While results for this special case follow by explicit calculation, the study of the asymptotic behavior of non-trivial flows has been an area of active research in recent years, where a guiding question has been to understand how robust this mechanism might be.

In this article, we construct a negative example in the form of a small sine wave perturbation to a linear shear

$$
U(y)=y+c \sin (y)
$$

with $|c|<\frac{1}{2}$. More precisely, for simplicity of calculation we consider the approximate system

$$
U(y)=y, \beta(y)=c \sin (y),
$$

which we introduce in Section 2. We thus omit a term $c \sin (y) \partial_{x}$ compared to the linearized Euler equations. Here we remark that in the setting of large period $L$ in $x$ and for small frequencies one may heuristically expect that $\partial_{x} \sim L^{-1}$ is negligible compared to $\partial_{x} \Delta^{-1} \sim L$. However, we do not establish error bounds on this approximation in this paper.

We stress that $U(y)$ is Bilipschitz and smooth and that $\beta(y)$ is small and analytic. Our choice of $U(y)=y$ is motivated by the existing linear and nonlinear damping results for Couette flow, where strict monotonicity and the associated shearing mechanism serve to stabilize the flow, but require either smallness conditions (see for example [CZZ19], [Zi116]) or non-resonance conditions (see for example [WZZ17]). More precisely, one needs to require that the linearized operator

$$
U(y) \partial_{x}+U^{\prime \prime}(y) \partial_{x} \Delta^{-1}
$$

has no embedding eigenvalues, which is not straightforward to check. As a related result on eigenvalue instabilities we mention the work of Lin [Lin03], who shows that if $U$ has a an inflection point $y_{s}$ inside an interval $I$ and

$$
K(y):=-\frac{U^{\prime \prime}(y)}{U(y)-U\left(y_{s}\right)}
$$

is positive and

$$
-\partial_{y}^{2}-K(y)
$$

has a negative eigenvalue, then linearized Euler equations on $\mathbb{R} \times I$ is exponentially unstable (that is, there exists an eigenvalue with positive real part). Furthermore, we mention that there has been much interest in the instability of Kolmogorov-type flows $U(y)=\cos (m y)$ with $m \in \mathbb{N}$ large, e.g. [FH98], for which $K(y)=m^{2}$ is constant. One aim of this article is then to understand how such an instability mechanism at the level of $U^{\prime \prime}$ may interact with shear and mixing by a monotone profile $U(y)$.

In view of the stability results exploiting convexity which we mentioned in the introduction, we note that there are special settings in which the shear profile is both convex and monotone and one can exploit both mechanisms. In the article of Bedrossian, Coti Zelati and Vicol [BCV17] on circular flows, monotone, convex profiles are considered and no smallness condition is required. However, we observe that convexity by itself is only sufficient given a beneficial sign. Indeed, in the sense of Arnold's theory Kolmogorov flow, $U(y)=\cos (y)$, is strictly convex with 
$\frac{U^{\prime \prime}}{U}=-1<0$, but is known to be nonlinearly stable only on a short torus $\mathbb{T}_{L} \times \mathbb{T}$, $L<1$ but unstable on a long torus $L \gg 1$, [MS61].

Our main question of this article concerns the robustness of the stabilization by shearing for Bilipschitz, non-convex profiles for short and long tori, in analogy to the results on Kolmogorov flow as a convex flow. Here, as in other works on (phase-)mixing [MV11, BMM16, BM15] it is useful to work in coordinates moving with the shear and consider the profile of the vorticity

$$
W(t, x, y):=\omega(t, x+t y, y) .
$$

As observed for instance in [LZ11, Theorem 3] and to some extent already in the classical work of Orr [Orr07], control of $W(t)$ in Sobolev regularity allows one to deduce asymptotic stability and damping of the velocity field by the estimate

$$
\begin{aligned}
\left\|v(t)-\int_{\mathbb{T}} v(t, x, \cdot) d x\right\|_{L^{2}} & \leq C(1+t)^{-1}\|W(t)\|_{H^{1}}, \\
\left\|v(t) \cdot e_{2}\right\|_{L^{2}} & \leq C(1+t)^{-2}\|W(t)\|_{H^{2}} .
\end{aligned}
$$

In this sense inviscid damping of the velocity field follows as consequence of the stability of the profile $W$. Our main results are summarized in the following theorem.

Theorem 1. Let $c \in\left(0, \frac{1}{2}\right)$ and consider the following linear problem

$$
\partial_{t} \omega+y \partial_{x} \omega+c \sin (y) \partial_{x} \Delta^{-1} \omega=0,
$$

on the domain $\mathbb{T}_{L} \times \mathbb{R}$ with initial data $\omega_{0}$. We consider this equation as a perturbation of the transport problem $\partial_{t}+y \partial_{x}$ (by a compact operator) and study the stability of the profile

$$
W(t, x, y):=\omega(t, x+t y, y)
$$

which moves with the shear dynamics.

Then if $c L<\frac{1}{2 \pi}$ it holds that for any $s \geq 0$ the evolution is stable in $H^{s}$ in the sense that there exists $C_{s}$ such that for any time $t \geq 0$

$$
\|W(t)\|_{H^{s}} \leq C_{s}\left\|\omega_{0}\right\|_{H^{s}} .
$$

Furthermore, there exists $C_{0}$ such that if the Fourier transform of $\omega_{0}$ satisfies

$$
\left\|\omega_{0}\right\|_{\mathcal{G}_{1, C}}^{2}:=\int \exp (C|\eta|)\left|\mathcal{F}_{y} \omega_{0}(\eta)\right|^{2}<\infty
$$

for some $C>C_{0}$, then also $\|W(t)\|_{\mathcal{G}_{1, C-C_{0}}}<\infty$ for all times.

On the other hand, suppose that $c<\frac{1}{10}$ and $L$ is such that $c \pi L>20$, but $\pi c^{2} L<1$ (e.g. choose $L \sim c^{-3 / 2}$ ). Then there exists smooth initial data $\omega_{0}$ (compactly supported in Fourier space), a frequency $k$ and $d>1$ such that its Fourier transform satisfies

$$
\left|\mathcal{F} W\left(t_{j}, k, \eta=k t_{j}\right)\right| \geq d^{t_{j}}
$$

for all $t_{j}=j \in \mathbb{N}$. That is, the evolution is exponentially growing along a sequence of times.

The growth mechanism in the second result here is not given by an eigenfunction construction, but given by a new cascade mechanism which propagates in the frequency $\eta$ associated to $y$. We stress that this resonance chain is distinct from the one considered in [DM18], [BM15] which propagates in the frequency $k$ associated to $x$ (see Section 3 ). In the regime of large period $L$ it is, to our knowledge, not known whether the problem (2) may additionally possess eigenvalue instabilities 
(for $\omega$ ). Our main aim in this article thus lies in establishing the cascade mechanism as a new, interesting instability/resonance mechanism, which also yields exponential growth (of $W$ ).

For simplicity of calculation and presentation we neglect the effects of boundaries and consider the setting of an infinite periodic channel $\mathbb{T}_{L} \times \mathbb{R}$. We however expect that our results should extend with moderate technical effort to the setting of smooth compactly supported perturbations to Couette flow (which is considered in [Jia19]) and to more general Bilipschitz flows as considered in [Zil16].

Our article is organized as follows:

- In Section 1.1 we recall some definitions and summarize notational conventions used throughout the article.

- In Section 2 we first establish global well-posedness of the equations and some rough upper growth bounds. Afterwards we establish the stability statement of Theorem 1 by means of a Duhamel iteration estimate. We further present a second proof by means of a Lyapunov functional, which is very transparent but requires stronger assumptions.

- In Section 3 we analyze the resonance mechanism in detail and how it can excite neighbors of resonant modes. As our main result we show that if $c L$ is sufficiently large this growth can be sustained, resulting in an exponential growth rate. In particular, this shows that it is not sufficient for a flow to be smooth and bilipschitz in order for asymptotic stability and linear inviscid damping to hold, but that some further smallness or non-resonance condition is required. While some eigenvalue instability constructions are available in the literature [WZZ18], we here present a new cascade mechanism underlying this sustained growth as well an explicit example.

Acknowledgments. Yu Deng acknowledges support by NSF grant DMS-1900251.

Christian Zillinger would like to thank the Max-Planck Institute for Mathematics in the Sciences where part of this work was written for its hospitality.

Christian Zillinger's research is supported by the ERCEA under the grant 014 669689-HADE and also by the Basque Government through the BERC 2014-2017 program and by Spanish Ministry of Economy and Competitiveness MINECO: BCAM Severo Ochoa excellence accreditation SEV-2013-0323.

1.1. Notation. In this article we are interested in the evolution of the vorticity in the linearized Euler equations around $v=(y+c \sin (y), 0)$ or more precisely a slightly simplified system (see Section 2 ) on $\mathbb{T}_{L} \times \mathbb{R}$.

As mentioned in Theorem 1 and as we discuss in Section 2, instead of considering $\omega$ in Eulerian (= Cartesian) coordinates it is useful to switch to Lagrangian coordinates and study the profile

$$
W(t, x, y)=\omega(t, x+t y, y) .
$$

We then study the Fourier transform of $W$ with respect to both $x$ and $y$, which we with slight abuse of notation again denote by $\omega$ :

$$
\omega(t, k, \eta)=\mathcal{F}_{x, y}(W)(t, k, \eta) .
$$

We further remark that, since the coefficient functions in our problem do not depend on $x$, the evolution equations decouple with respect to $k \in \frac{2 \pi}{L} \mathbb{Z}$. For this reason we may consider $k$ to arbitrary but fixed and suppress the $k$-dependence in our notation by writing $\omega(t, \eta)$. 
As we discuss in Remark 2 and in Section 3, due to the structure of $\sin (y)$, the evolution of $\omega(t, \eta)$ only depends on $\omega\left(t, \eta^{\prime}\right)$ if $\eta-\eta^{\prime} \in \mathbb{Z}$ is an integer. Thus, while the Fourier variable $\eta \in \mathbb{R}$ naturally belongs to the whole space, in Section 3 we restrict to discrete classes $\eta_{0}+\mathbb{Z}=\left(\eta_{0}-\left\lfloor\eta_{0}\right\rfloor\right)$ with $\eta_{0}-\left\lfloor\eta_{0}\right\rfloor \in[0,1)$. For simplicity of notation we here write results for the specific case $\eta_{0}-\left\lfloor\eta_{0}\right\rfloor=0$, so that $\eta \in \mathbb{Z}$.

\section{Global Well-posedness and Duhamel Iterations}

The linearized 2D Euler equations around $v=(y+c \sin (y), 0)$ are given by:

$$
\partial_{t} \omega+(y+c \sin (y)) \partial_{x} \omega+c \sin (y) v_{2}=0 .
$$

As the map $\omega \mapsto v_{2}=\partial_{x} \Delta^{-1} \omega$ is compact, we consider the transport by the linear profile to be dominant, change to Lagrangian coordinates $(x+t y, y)$ and consider

$$
W(t, x, y)=: \omega(t, x+t y, y)
$$

With respect to these coordinates the equation is given by

$$
\begin{aligned}
\partial_{t} W+c \sin (y) \partial_{x} W+c \sin (y) \partial_{x} \Delta_{t}^{-1} W & =0 \\
\Delta_{t} & :=\partial_{x}^{2}+\left(\partial_{y}-t \partial_{x}\right)^{2} .
\end{aligned}
$$

Here, our choice of coordinates has the benefit of the very transparent structure of $\Delta_{t}^{-1}$ as a Fourier multiplier. As the coefficient functions do not depend on $x$, we note that this system decouples in the associated frequency $k$, which we hence treat as arbitrary but fixed. Since our main focus is on considering the effect of long tori (and thus very small frequency $k$ ), we further consider an approximate system

$$
\partial_{t} W+c \sin (y) \partial_{x} \Delta_{t}^{-1} W=0,
$$

where we omit the contribution by $c \sin (y) \partial_{x}$ and with slight abuse of notation denote the Fourier transform of $W$ by $\omega$ again.

This then leads to the following system:

$$
\partial_{t} \omega(\eta)+\frac{c}{2} \frac{k}{k^{2}+(\eta+1-k t)^{2}} \omega(\eta+1)-\frac{c}{2} \frac{k}{k^{2}+(\eta-1-k t)^{2}} \omega(\eta-1)=0 .
$$

Since the system decouples in $k$ and leaves the mode $k=0$ invariant, in the following we without loss of generality consider $k \neq 0$ and equivalently rescale time as $\tau=k t$, which yields:

$$
\partial_{\tau} \omega(\eta)+\frac{c}{2} \frac{1}{k^{2}+(\eta+1-\tau)^{2}} \omega(\eta+1)-\frac{c}{2} \frac{1}{k^{2}+(\eta-1-\tau)^{2}} \omega(\eta-1)=0 .
$$

Remark 2. This equation is an ODE system with nearest-neighbor interaction. In particular, since modes interact only via chains of neighbors, the system decouples into problems on $\eta \in \eta^{*}+\mathbb{Z}$, with $\eta^{*} \in[0,1)$. Considering $\eta^{*}$ as arbitrary but fixed and with slight abuse of notation shifting time by $\eta^{*}$ to prescribe "initial" data at that time it thus suffices to consider the periodic problem $\eta \in \mathbb{Z}$. We remark that this setting would also appear when considering periodic perturbations with respect to Lagrangian coordinates $(x+t y, y)$, which seems physically unmotivated, but by the above consideration appears naturally due to the decoupling structure.

Roughly estimating the coefficient functions by $\frac{c}{2 k^{2}}$, we immediately obtain the following suboptimal global well-posedness result. 
Lemma 3. Let $X=L^{2}\left(\rho, \frac{2 \pi}{L} \mathbb{Z} \times \mathbb{R}\right)$ be a weighted $L^{2}$ space in Fourier space such that its weight satisfies

$$
\sup _{k, \eta} \frac{\rho(k, \eta \pm 1)}{\rho(k, \eta)} \leq C_{1}<\infty
$$

This for example includes fractional Sobolev spaces $H^{s}$ or Gevrey spaces.

Then there exists a constant $C$ such that the solution $\omega$ of (5) satisfies

$$
\|\omega(\tau)\|_{X} \leq \exp \left(C c L^{2} \tau\right)\left\|\omega_{0}\right\|_{X}
$$

for all $\tau \geq 0$.

We remark that the time variable $\tau$ includes a factor $L$ and that expressed with respect to $t$ the growth factor is given by $\exp (C c L t)$.

Proof. We note that

$$
\left\|\partial_{\tau} \omega\right\|_{X} \leq\left\|c \frac{1}{k^{2}+(\eta \pm 1-\tau)^{2}} \omega(\eta \pm 1)\right\|_{X} \leq c C L^{2}\|\omega\|_{X} .
$$

The result then immediately follows by integrating the differential inequality.

While this a priori bound is sufficient to establish global well-posedness, it is far from sharp. Indeed, the following theorem shows that for $c L$ sufficiently small, the evolution is globally stable in a Lyapunov sense. In contrast, in Theorem 9 we show that if $L \gg c^{-1}$, then this stability fails and the evolution is exponentially unstable. Here, we stress that the shear profile is Bilipschitz and $c \sin (y)$ is smooth and bounded. Furthermore, we show that the described growth is due to a new cascade mechanism, where chains of resonances excite higher and higher modes in $y$ (while the nonlinear echo chain mechanism excites smaller and smaller modes in $x$ and stops at mode 1 ).

As first result, we consider the setting of very small $c$, which is a amenable to the construction of a Lyapunov functional as in [CZZ19]. Here, the simple Fourier structure allows for a particularly transparent proof. In a second Theorem 8 we introduce a different method of proof by expressing the Duhamel iteration as formal infinite series of integrals over paths (see Definition 6).

Theorem 4 (Global stability for small $c L$ ). Let $j \in \mathbb{N}$ and consider the stability problem for the pde (5) on $\mathbb{T}_{L} \times \mathbb{R}$ in $H^{j}$. Then there exists $C_{0}=C_{0}(j)>0$ such that if $c L<C_{0}$, there exists a constant $C$ such that

$$
\|\omega(\tau)\|_{H^{j}} \leq C\left\|\omega_{0}\right\|_{H^{j}}
$$

for all $\tau \geq 0$. Furthermore, $\partial_{x} \Delta_{t}^{-1} W \in L_{t}^{2} H^{j}$ and $\omega$ converges strongly in $H^{j}$ as $\tau \rightarrow \infty$.

Proof. We define the Fourier weight

$$
a(\tau, \eta)=\exp \left(C_{1} c \arctan \left(C_{2}(\eta-\tau)\right)\right.
$$

with $C_{1}, C_{2}>0$ to be fixed later. 
Then it holds that

$$
\begin{aligned}
& \frac{d}{d \tau} a(\tau, \eta)|\omega(\tau, \eta)|^{2} \\
= & -C_{1} C_{2} \frac{c}{1+C_{2}^{2}(\eta-\tau)^{2}} a(\tau, \eta)|\omega(\tau, \eta)|^{2} \\
& +2 a(\tau, \eta) \frac{c}{k^{2}+(\eta+1-\tau)^{2}} \omega(\tau, \eta+1) \omega(t, \eta) \\
& -2 a(\tau, \eta) \frac{c}{k^{2}+(\eta-1-\tau)^{2}} \omega(\tau, \eta-1) \omega(\tau, \eta) .
\end{aligned}
$$

We may estimate $|\omega(\tau, \eta \pm 1) \omega(\tau, \eta)| \leq \frac{1}{2}\left(|\omega(\tau, \eta \pm 1)|^{2}+|\omega(\tau, \eta)|^{2}\right)$ and integrate over all $\eta$ to obtain:

$$
\begin{aligned}
& \frac{d}{d \tau} \int_{\eta} a(\tau, \eta)|\omega(t, \eta)|^{2} \\
\leq & -C_{1} C_{2} \int_{\eta} \frac{c}{1+C_{2}^{2}(\eta-t)^{2}} a(\tau, \eta)|\omega(\tau, \eta)|^{2} \\
& +\int_{\eta} a(\tau, \eta)\left(\frac{c}{k^{2}+(\eta+1-t)^{2}}+\frac{c}{k^{2}+(\eta-1-t)^{2}}\right)|\omega(\tau, \eta)|^{2} \\
& +\int_{\eta}(a(\tau, \eta-1)+a(\tau, \eta+1)) \frac{c}{k^{2}+(\eta-\tau)^{2}}|\omega(\tau, \eta)|^{2},
\end{aligned}
$$

where we shifted $\eta$ in the last sum. By the definition of our weight $a$ it holds that

$$
(a(\tau, \eta-1)+a(\tau, \eta+1)) \leq \exp \left(2 C_{1} c\right) a(\tau, \eta)
$$

and that

$$
\left(\frac{c}{k^{2}+(\eta+1-\tau)^{2}}+\frac{c}{k^{2}+(\eta-1-\tau)^{2}}\right) \leq 4 k^{-2} \frac{c}{1+k^{-2}(\eta-\tau)^{2}} .
$$

Hence, we may conclude that

$$
\frac{d}{d \tau} \int_{\eta} a(\tau, \eta)|\omega(\tau, \eta)|^{2} \leq-\frac{C}{2} \int_{\eta} \frac{c}{k^{2}+(\eta-\tau)^{2}} a(\tau, \eta)|\omega(\tau, \eta)|^{2} \leq 0,
$$

provided that

$$
\begin{aligned}
C_{2} & =k^{-1}, \\
C_{1} C_{2}-2 k^{-2} \exp \left(2 C_{1} c\right) & \geq \frac{C}{2} .
\end{aligned}
$$

We may then fix $C_{2}=k^{-1} \leq L, C_{1}=4 k^{-1}$, at which point the estimate reduces to $4-2 \exp (2 c L)=: C / 2>0$, which is satisfied if $c L$ is sufficiently small.

We note that $(6)$ implies $L^{2}$ stability, since $a(\tau, \eta) \approx 1$. Furthermore, as $\left.\int_{\eta} a(\tau, \eta)|\omega(t, \eta)|^{2}\right|_{t=0} ^{T}$ is bounded, it follows that

$$
\int_{\eta} \frac{c}{k^{2}+(\eta-t)^{2}} a(\tau, \eta)|\omega(\tau, \eta)|^{2}
$$

is integrable in time. Expressing the evolution equation in integral form then further yields the claimed asymptotic stability in $L^{2}$.

It remains to establish stability in higher Sobolev norms. We define

$$
a_{j}(\tau, \eta):=\langle\eta\rangle^{j} a(\tau, \eta)
$$


for $j \in \mathbb{N}$ and further define

$$
A_{j}(\tau, \eta):=a_{j}(\tau, \eta)+\int_{j^{\prime} \leq j-1}\left(\begin{array}{l}
j \\
j^{\prime}
\end{array}\right) A_{j^{\prime}}(\tau, \eta) .
$$

Using (6) as the start of a proof by induction, we then claim that

$$
\frac{d}{d \tau} \int_{\eta} A_{j}(\tau, \eta)|\omega(\eta)|^{2} \leq-\frac{C}{2} \int_{\eta} \frac{c}{k^{2}+(\eta-\tau)^{2}} A_{j}(\tau, \eta)|\omega(\eta)|^{2} .
$$

Let thus $\hat{j} \geq 1$ be given and suppose that (9) is satisfied for all $j \leq \hat{j}$. Then it holds that

$$
\begin{aligned}
& \frac{d}{d t} \int_{\eta} A_{\hat{j}+1}(\tau, \eta)|\omega(\eta)|^{2} \\
= & \frac{d}{d t} \int_{\eta} a_{\hat{j}+1}(\tau, \eta)|\omega(\eta)|^{2}+2 \frac{d}{d t} \int_{\eta} A_{\hat{j}}(\tau, \eta)|\omega(\eta)|^{2} \\
\leq & -C \int_{\eta} \frac{c}{1+(\eta-\tau)^{2}} a_{\hat{j}+1}(\tau, \eta)|\omega(\eta)|^{2} \\
& +2 \int_{\eta} a_{\hat{j}+1}(\tau, \eta) \omega(\tau, \eta) \frac{d}{d \tau} \omega(\tau, \eta) \\
- & C \int_{\eta} \frac{c}{1+(\eta-\tau)^{2}} A_{\hat{j}}(\tau, \eta)|\omega(\eta)|^{2} .
\end{aligned}
$$

Plugging in the equation for $\frac{d}{d t} \omega(\tau, \eta)$ and using Young's inequality, we again have to control shifts:

$$
\begin{aligned}
a_{\hat{j}+1}(\tau, \eta \pm 1) & =\langle\eta \pm 1\rangle^{\hat{j}+1} a(\tau, \eta \pm 1) \\
& \leq \frac{a(\tau, \eta \pm 1)}{a(\tau, \eta)} a_{\hat{j}+1}(\tau, \eta)+\left(\langle\eta \pm 1\rangle^{\hat{j}+1}-\langle\eta\rangle^{\hat{j}+1}\right) a(\tau, \eta \pm 1) \\
& \leq \exp (c C) a_{\hat{j}+1}(\tau, \eta)+\int_{j^{\prime} \leq \hat{j}}\left(\begin{array}{c}
j \\
j^{\prime}
\end{array}\right) a_{j^{\prime}}(\tau, \eta \pm 1),
\end{aligned}
$$

where the binomial factors are obtained by expanding $\langle\eta \pm 1\rangle^{\hat{j}+1}-\langle\eta\rangle^{\hat{j}+1}$. As in the case $\hat{j}=0, \exp (c C) a_{\hat{j}+1}(\tau, \eta)$ may be absorbed by $\frac{d}{d \tau} a_{\hat{j}+1}(\tau, \eta)$ provided $c$ is sufficiently small. The additional correction by $\left(\begin{array}{c}j \\ j^{\prime}\end{array}\right) a_{j^{\prime}}(\tau, \eta \pm 1)$ is of lower order and can be absorbed into

$$
-C \int_{\eta} \frac{c}{k^{2}+(\eta-\tau)^{2}} A_{\hat{j}}(\tau, \eta)|\omega(\eta)|^{2}
$$

While the preceding method of proof allows for a useful control of the evolution and stability and is very explicit, it is only applicable in the regime of very small $c L$ and does not allow for a more precise description of the evolution in terms of the initial data.

In the following we hence instead argue by considering iterated Duhamel iterations as a formal infinite series. We remark that in [DZ19] we employed similar methods to study the (nonlinear) echo chain mechanism.

Lemma 5 establishes the convergence of the Duhamel iteration under very mild assumptions. In the following we then relate the Duhamel iteration to considering sums over paths (see Definition 6). Theorem 8 serves to introduce our new techniques 
in a transparent and accessible manner, also for $c$ small. Afterwards, in Theorem 9 we show that for $c$ larger than this, but still smaller than 0.5 , a cascade of resonances yields exponential growth and a well-described non-trivial asymptotic behavior.

Lemma 5. Let $U(y)$ be a measurable function and let $\beta \in L^{\infty}$. Consider the problem

$$
\partial_{t} \omega+\beta(y) \partial_{x} \tilde{\Delta}_{t}^{-1} \omega=0
$$

where

$$
\tilde{\Delta}_{t}=\partial_{x}^{2}+\left(\partial_{y}-t U^{\prime}(y) \partial_{x}\right)^{2}
$$

is obtained by conjugating $\Delta$ with the flow with $(U(y), 0)$. Since the equations preserve the $x$ average, additionally assume without loss of generality that $\int \omega_{0} d x=0$. Then there exists a constant $C$ possibly depending on the domain such that for all times $t \geq 0$ it holds that

$$
\|\omega(t)\|_{L^{2}}^{2} \leq e^{C\|\beta\|_{L^{\infty} t}}\left\|\omega_{0}\right\|_{L^{2}}^{2} .
$$

Furthermore, the formal infinite Duhamel series

$$
\begin{array}{r}
I d+\int_{0}^{t} \beta(y) \partial_{x} \tilde{\Delta}_{t_{1}}^{-1} d t_{1} \\
+\int_{0}^{t} \int_{0}^{t_{1}} \beta(y) \partial_{x} \tilde{\Delta}_{t_{1}}^{-1} \beta(y) \partial_{x} \tilde{\Delta}_{t_{2}}^{-1} d t_{2} d t_{1}+\ldots
\end{array}
$$

is convergent in the $L^{2}$ operator topology with the $j^{\text {th }}$-term bounded by $\frac{\left(C\|\beta\|_{L} \infty t\right)^{j}}{j !}$.

We point out that this lemma imposes very mild assumptions on $U$ and $\beta$. A big question in the following will be how far from optimal this estimate is when considering the specific case (5).

Proof of Lemma 5. We remark that the equation (10) is obtained from the linearized Euler equations (and related models where $\beta(y) \neq U^{\prime \prime}(y)$ )

$$
\partial_{t} \omega+U(y) \partial_{x} \omega+\beta(y) \partial_{x} \Delta^{-1} \omega=0,
$$

by considering Lagrangian coordinates

$$
(x+t U(y), y) .
$$

We observe that (12) and hence also equation (10) preserves the $x$-average of $\omega$ and therefore without loss of generality restrict to considering solutions whose $x$-average vanishes.

As the change to Lagrangian coordinates is an $L^{2}$ isometry, we observe that the $L^{2}$ operator norm of $\partial_{x} \tilde{\Delta}_{t}^{-1}$ does not depend on time. More precisely, by the above discussion we consider the operator norm of $\partial_{x} \Delta^{-1}$ restricted to functions with vanishing $x$-average, which we denote by the projection $P_{\neq}$on frequencies $k \neq 0$. Then $\partial_{x} \Delta^{-1} P_{\neq}$is a bounded operator, since the corresponding Fourier multiplier $\frac{i k}{k^{2}+\eta^{2}} 1_{k \neq 0}$ is uniformly bounded for all $k \in \mathbb{Z}, \eta \in \mathbb{R}$. Thus, the $L^{2}$ norm of $\beta(y) \partial_{x} \tilde{\Delta}_{t}^{-1} \omega(t)$ can be controlled by

$$
\|\beta\|_{L^{\infty}}\left\|\partial_{x} \Delta^{-1} P_{\neq}\right\|_{L^{2} \rightarrow L^{2}}\|\omega(t)\|_{L^{2}} .
$$

The exponential growth bound hence follows by Gronwall's lemma. 
In order to prove the convergence of the Duhamel series we need a more detailed description. We observe that $\omega$ solves the integral equation

$$
\omega(t)=\omega_{0}+\int_{0}^{t} \beta(y) \partial_{x} \tilde{\Delta}_{t_{1}}^{-1} \omega\left(t_{1}\right) d t_{1}
$$

Thus, repeatedly inserting this characterization we formally obtain

$$
\begin{aligned}
\omega(t)= & \omega_{0}+\int_{0}^{t} \beta(y) \partial_{x} \tilde{\Delta}_{t_{1}}^{-1} \omega_{0} d t_{1} \\
& +\int_{0}^{t} \int_{0}^{t_{1}} \beta(y) \partial_{x} \tilde{\Delta}_{t_{1}}^{-1} \beta(y) \partial_{x} \tilde{\Delta}_{t_{2}}^{-1} \omega_{0} d t_{2} d t_{1} \\
& +\int_{0}^{t} \int_{0}^{t_{1}} \int_{0}^{t_{2}} \ldots d t_{3} d t_{2} d t_{1}+\ldots
\end{aligned}
$$

It remains to be shown that the series on the right-hand-side is well-defined and convergent.

As remarked above, due to the decoupling structure of the equation with respect to the frequencies in $x$, we may without loss of generality establish such a result for $\omega_{0}$ given by a single ( $y$-dependent) mode $k$ (with estimate uniform in $k$, of course). The key property we now use is that $\beta(y) \partial_{x} \tilde{\Delta}_{t}^{-1}$ depends on time only due to the change of variables $(t, x, y) \mapsto(t, x+t U(y), y)$. For any single mode data at frequency $k$, this change of variables in turn corresponds to a multiplication by $e^{i k t U(y)}$.

Let thus $k \neq 0$ be arbitrary but fixed and let $G_{1}\left(y, y^{\prime}\right)$ denote the Green's function of $\partial_{x} \Delta^{-1}=i k\left(-k^{2}+\partial_{y}^{2}\right)^{-1}$ (that is, the time $t=0$ case). Then the kernel of $\partial_{x} \tilde{\Delta}_{t}^{-1}$ is given by $e^{i k t U(y)} G_{1}\left(y, y^{\prime}\right) e^{-i k t U\left(y^{\prime}\right)}$ and we may isolate the time-dependence of the kernel of

$$
\beta(y) \partial_{x} \tilde{\Delta}_{t}^{-1}
$$

as

$$
e^{-i k t U(y)} \beta(y) G_{1}\left(y, y^{\prime}\right) e^{i k t U\left(y^{\prime}\right)}=: e^{i k t\left(U(y)-U\left(y^{\prime}\right)\right)} K\left(y, y^{\prime}\right) .
$$

The $j$-th term of the Duhamel iteration is thus given by

$$
\int_{0}^{t} \int_{0}^{t_{1}} \cdots \int_{0}^{t_{j-1}} \iint_{y_{1}, \ldots y_{j}} \prod e^{i k t\left(U\left(y_{j-1}\right)-U\left(y_{j}\right)\right)} K\left(y_{j-1}, y_{j}\right) .
$$

We now can bound all the exponentials by 1 and may estimate this integral as follows:

$$
\frac{t^{j}}{j !} \iint_{y_{1}, \ldots y_{j}} \prod\left|K\left(y_{j-1}, y_{j}\right)\right|
$$

Recalling the structure of $K\left(y, y^{\prime}\right)$ as $c_{k} e^{-\left|k\left(y-y^{\prime}\right)\right|}$ in the whole space or a function in terms of sinh and cosh in the case of an interval, we note that also $\left|K\left(y, y^{\prime}\right)\right|$ induces an operator with finite operator norm and that we thus obtain a bound in operator norm by

$$
\frac{t^{j}}{j !} C^{j}
$$

where $C$ is the operator norm associated to $\left|K\left(y, y^{\prime}\right)\right|$. The result hence follows by comparison with the exponential series. 
With this abstract convergence result at hand, we next establish a finer description of each Duhamel iteration and the resulting value of the infinite series. Here we use that our equation only contains nearest-neighbor interaction and that the $j$-th Duhamel iteration thus only relates modes that are at most $j$ apart. We hence show that all non-trivial contributions from initial data at a mode $\left(k, \eta_{0}\right)$ to a mode $\left(k, \eta_{1}\right)$ correspond to paths from one mode to the other. Furthermore, when considering the infinite Duhamel series, the integrals are given solely in terms of the initial data.

Definition 6 (Path). A path $\gamma$ from $\eta_{0} \in \mathbb{R}$ to $\eta_{1} \in \mathbb{R}$ is a sequence $\gamma=$ $\left(\gamma_{0}, \gamma_{1}, \gamma_{2}, \ldots, \gamma_{j}\right)$ with $\gamma_{0}=\eta_{0}, \gamma_{j}=\eta_{1}$ and $\left|\gamma_{i+1}-\gamma_{i}\right|=1$. We call $|\gamma|:=j$ the length of $\gamma$.

Given two times $t_{0}<t_{1}$ we then associate to each path $\gamma$ a Duhamel integral $I_{\gamma}\left[t_{0}, t_{1}\right]:$

$$
I_{\gamma}\left[t_{0}, t_{1}\right]=\iint_{t_{0} \leq \tau_{0} \leq \tau_{1} \leq \cdots \leq \tau_{j} \leq t_{1}} \omega\left(t_{0}, k, \gamma_{0}\right) \prod_{i=0}^{|\gamma|-1} \operatorname{sgn}\left(\gamma_{i+1}-\gamma_{i}\right) \frac{c}{k^{2}+\left(\gamma_{i}-\tau_{i}\right)^{2}} .
$$

The sign of $\gamma_{i+1}-\gamma_{i}$ here accounts for the different signs of the exponentials in $2 i \sin (y)=e^{i y}-e^{-i y}$. We note that paths between $\eta_{0}$ and $\eta_{1}$ can only exist if $\eta_{0}-\eta_{1} \in \mathbb{Z}$ is an integer. As discussed in Remark 2 this due to the fact that products of $\sin (y)$ introduce integer-sized Fourier shifts and the evolution thus decouples into equivalence classes

$$
\xi+\mathbb{Z}
$$

with $\xi \in[0,1)$. As remarked in Section 1.1 we in the following for simplicity of notation consider $\xi=0$ and thus consider $\eta \in \mathbb{Z}$ discrete. In particular, we naturally associate this setting with $y \in \mathbb{T}$ and hence $\omega(t) \in L^{2}\left(\mathbb{T}_{L} \times \mathbb{T}\right)$.

With this definition and conventions we may restate the Duhamel characterization of a solution in Lemma 5 as a series of paths. Here we recall from Remark 2 that the evolution decouples into classes $[\eta] \in \mathbb{R} / \mathbb{N}$ and that hence $\omega(t, k, \eta)$ depends only

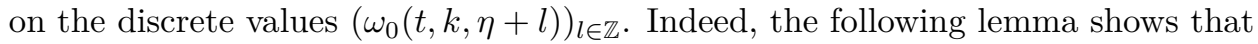
$\omega(t, k, \eta)$ can be computed in terms of a sum over paths ending in $\eta$. For simplicity of notation in the formulation of this lemma we use the discrete structure to phrase this a result for $\omega_{0} \in L^{2}(\mathbb{T} \times \mathbb{T})$ (that is $\left.\eta \in \mathbb{Z}\right)$. The case $\omega_{0} \in L^{2}(\mathbb{T} \times \mathbb{R})$ is then obtained by studying all classes $\mathbb{R} / \mathbb{Z}$.

Lemma 7. Let $k$ be arbitrary but fixed and let $\eta_{0}$ be given. Let further $\omega_{0} \in$ $L^{2}\left(\mathbb{T}_{L} \times \mathbb{T}\right)$ with $\mathcal{F}\left(\omega_{0}\right)(k, \eta)=\alpha \delta_{\left(k_{0}, \eta_{0}\right)}(k, \eta)$. Then for any time $t>0$ and any frequency $\left(k_{0}, \eta\right)$ it holds that the solution $\omega(t)$ satisfies

$$
\omega(t, k, \eta)=\omega_{0}(k, \eta)+\sum_{\gamma: \gamma_{0}=\eta_{0}, \gamma_{|\gamma|}=\eta} I_{\gamma}[0, t] .
$$

Proof. We recall that by Lemma 5 it holds that (in an $L^{2}$ sense)

$$
\begin{aligned}
\omega(t, x, y)= & \omega_{0}(x, y)+\int_{0}^{t} \sin (y) \partial_{x} \Delta_{t_{1}}^{-1} \omega_{0} d t_{1} \\
& +\iint_{0<t_{1}<t_{2}<t} \sin (y) \partial_{x} \Delta_{t_{2}}^{-1} \sin (y) \partial_{x} \Delta_{t_{1}}^{-1} \omega_{0} d t_{1} d t_{2}+\ldots
\end{aligned}
$$

where

$$
\Delta_{t}=\partial_{x}^{2}+\left(\partial_{y}-t \partial_{x}\right)^{2}
$$


We may then again use that this evolution decouples with respect to the frequency $k$ in $x$ to replace all operators $\partial_{x}$ by a multiplication by $i k$. Furthermore, we note that $2 i \sin (y)=e^{i y}-e^{-i y}$ is a sum of (Fourier) shifts in $\eta$ and we can hence identify

$$
\begin{array}{r}
\mathcal{F}_{x, y}\left(\int_{0}^{t} \sin (y) \partial_{x} \Delta_{t_{1}}^{-1} \omega_{0} d t_{1}\right)(k, \eta) \\
=\int_{0}^{t} \frac{1}{2 i} \frac{i k}{k^{2}+((\eta-1)-k t)^{2}}\left(\mathcal{F} \omega_{0}\right)(k, \eta-1) d t_{1} \\
-\int_{0}^{t} \frac{1}{2 i} \frac{i k}{k^{2}+((\eta+1)-k t)^{2}}\left(\mathcal{F} \omega_{0}\right)(k, \eta+1) d t_{1}
\end{array}
$$

as corresponding to the two paths $(\eta-1, \eta)$ and $(\eta+1, \eta)$. Analogously, for any $j \in \mathbb{N}$ we may identify the $j$-th Duhamel integral as the sum over $2^{j}$ paths of length $j$. Since we without loss of generality assumed that $\omega_{0}(t, k, \xi)$ is only non-zero for a single $\xi \in \mathbb{Z}, \xi=\eta_{0}$, in order to compute $\omega(t, k, \eta)$ we then only need to take into account those paths $\gamma$ which start in $\eta_{0}$ and end in $\eta$. Thus, for each fixed $\eta$, we obtain

$$
\begin{aligned}
\omega(t, k, \eta) & =\omega_{0}(k, \eta)+\sum_{j} \sum_{\gamma:|\gamma|=j, \gamma_{0}=\eta_{0}, \gamma_{|\gamma|}=\eta} I_{\gamma}[0, t] \\
& =\omega_{0}(k, \eta)+\sum_{\gamma: \gamma_{0}=\eta_{0}, \gamma_{|\gamma|}=\eta} I_{\gamma}[0, t] .
\end{aligned}
$$

We remark that any initial datum $\left(\mathcal{F} \omega_{0}(k, \eta)\right)_{\eta \in \mathbb{Z}} \in \ell^{2}(\mathbb{Z})$ can be decomposed into a sum of $\delta_{\eta}$ 's and the result for general initial data hence immediately follows by linearity. Furthermore, we may repeat the same proof for starting times other than 0 .

Theorem 8 (Second stability theorem). Suppose that $c L 2 \pi<1$ and let $\omega_{0} \in$ $L^{2}\left(\mathbb{T}_{L} \times \mathbb{T}\right)$. Then for all $\tau \geq 0$ and all $\eta$ it holds that

$$
\left|\omega(\tau, \eta)-\omega_{0}(\eta)\right| \leq \frac{1}{1-c L 2 \pi} \sum_{\eta_{0} \in \mathbb{Z}}(c L 2 \pi)^{\left|\eta-\eta_{0}\right|}\left|\omega_{0}\left(\eta_{0}\right)\right| .
$$

We remark that the right-hand side corresponds to a convolution in $\eta_{0}$ against an exponentially decreasing function. In particular, this implies that the evolution is stable in $H^{s}$ for any $s>0$ and in Gevrey regularity.

Proof. By our assumption on $c$ it holds that

$$
\int_{\mathbb{R}} \frac{c}{k^{2}+\tau^{2}} d \tau=\frac{c}{|k|} \pi=: d<\frac{1}{2} .
$$

In contrast to the norm-based approached of Theorem 4 and Lemma 3, we in the following consider the frequency-wise evolution of the solution established in Lemma 7. Then (13) will allow us to bound each integral $I_{\gamma}\left[t_{0}, t_{1}\right]$ in terms of $d^{|\gamma|}$.

Suppose at first that $\omega_{0}=\delta_{\eta_{0}}$ and fix a time $\tau>0$. Then by Lemma 7 the value of $\omega(\eta)$ at time $\tau$ can be obtained by summing over all integrals corresponding to 
paths starting in $\eta_{0}$ and ending in $\eta$ :

$$
\omega(\tau, \eta)-\omega(0, \eta)=\sum_{\gamma: \gamma_{0}=\eta_{0}, \gamma_{|\gamma|}=\eta} I_{\gamma}[0, \tau] .
$$

By Fubini's theorem and (13) we may easily estimate each integral by

$$
\left|I_{\gamma}[0, \tau]\right| \leq d^{|\gamma|} .
$$

Now for any given length $j$ there are only $2^{j}$ paths starting in $\eta_{0}$ of length $|\gamma|=j$ (of which only a fraction ends in $\eta$ ). If we denote the length of the shortest path connecting $\eta_{0}$ and $\eta$ by $\operatorname{dist}\left(\eta_{0}, \eta_{1}\right)$ it thus follows that

$$
\begin{aligned}
|\omega(\tau, \eta)-\omega(0, \eta)| & \leq \sum_{j \geq \operatorname{dist}\left(\eta_{0}, \eta_{1}\right)}(2 d)^{j} \\
& =\frac{1}{1-2 d}(2 d)^{\operatorname{dist}\left(\eta_{0}, \eta_{1}\right)} .
\end{aligned}
$$

Having established the bound for the special case $\omega_{0}=\delta_{\eta_{0}} \in \ell^{2}(\mathbb{Z})$, we now turn to the general case $\omega_{0} \in \ell^{2}(\mathbb{Z})$. Then we may decompose $\omega_{0}$ as

$$
\omega_{0}=\sum_{\eta_{0}} \omega_{0}\left(\eta_{0}\right) \delta_{\eta_{0}}
$$

and may use the linearity of the problem to compute

$$
\omega(\tau, \eta)-\omega(0, \eta)=\sum_{\eta_{0}} \omega_{0}\left(\eta_{0}\right) \sum_{\gamma: \gamma_{0}=\eta_{0}, \gamma_{|\gamma|}=\eta} I_{\gamma}[0, \tau] .
$$

Therefore, by the triangle inequality it follows that

$$
|\omega(\tau, \eta)-\omega(0, \eta)| \leq \frac{1}{1-2 d} \sum_{\eta_{0}}(2 d)^{\left|\eta-\eta_{0}\right|}\left|\omega_{0}\left(\eta_{0}\right)\right|,
$$

which is a (discrete) convolution in $\eta_{0}$.

\section{Instability for Long Tori And Cascades}

As a complementary result to the stability established in Section 2, we show that for long tori or respectively slightly larger $c$ the dynamics are exponentially unstable. Here, we do not construct eigenfunctions but instead establish a new cascade mechanism for resonances with respect to the frequency in $y$. We recall that by the Orr mechanism the multiplier associated with the stream function

$$
\frac{1}{k^{2}+(\eta-k t)^{2}}
$$

is largest when $\eta-k t=0$. In the study of the nonlinear problem [DM18, BM15, DZ19] this resonance underlies the main growth mechanism, where $\eta$ is roughly fixed and a mode $k$ at time $t \approx \frac{\eta}{k}$ excites a mode $k-1$, which then later excites a mode $k-2$ and so on. As we discuss in [DZ19] this cascade is a property of the linearized problem around the low-frequency part of the vorticity depending on $x$, e.g. $c \sin (x)$. As one of the main results of this paper we show that the linearized problem around the $x$-independent part, i.e. the shear flow component, also exhibits a cascade mechanism but with respect to $\eta$. That is, here $k$ is fixed and a mode $\eta$ at time $t \approx \frac{\eta}{k}$ excites the mode $\eta+1$, which later excites the mode $\eta+2$ at time $t \approx \frac{\eta+1}{k}$ and so on. 
We in particular note the following similarities and differences, where we refer to the cascades as $k$-chain and $\eta$-chain, respectively.

- In the $k$-chain for any initial mode $\left(k_{0}, \eta_{0}\right)$ the resonant times are given by $t_{k}=\frac{\eta_{0}}{k}$ with $k=k_{0}, k_{0}-1, \ldots, 1$. In particular, starting from any strictly positive time, there are only finitely many resonances and no resonances after a maximal time $t=\eta$. In contrast, the $\eta$-chain has the resonant times $t_{\eta}=\frac{\eta}{k_{0}}$ with $\eta=\eta_{0}, \eta_{0}+1, \ldots$, which is a cascade of infinite length.

- The sequences of resonant times $t_{k}$ is unevenly spaced, while the sequence of times $t_{\eta}$ is a rescaled integer sequence.

- The total growth exhibited by a $k$-chain is given by

$$
\frac{c \eta_{0}}{k_{0}^{2}} \frac{c \eta_{0}}{\left(k_{0}-1\right)^{2}} \cdots=\frac{\left(c \eta_{0}\right)^{k_{0}}}{\left(k_{0} !\right)^{2}}
$$

which attains its maximum with respect to $k_{0}, \exp \left(\sqrt{c \eta_{0}}\right)$ for $k_{0} \approx \sqrt{c \eta_{0}}$. This growth factor corresponds to a Gevrey regularity class.

The growth by the $\eta$-chain in contrast is exponential in time and tied to the $x$ frequency of the perturbation $k_{0}$ instead of the regularity. That is, the growth factor is given by

$$
\left(2 \pi \frac{c}{k}\right)^{t}
$$

provided $2 \pi \frac{c}{k} \gg 1$, even if we start with single mode initial data at $\eta=0$.

- As can be seen from the growth factors and as shown in the previous section there is no growth in the $\eta$-chain if $\frac{c}{k}$ is small. Similarly, there is only growth by a constant in the $k$-chain if $\sqrt{c \eta_{0}}$ is small.

Theorem 9 (Instability). Let $0<c_{0}<\frac{1}{10}$, then there exists $L$ such that for any $c>c_{0}$ the evolution (5) is exponentially unstable in $L^{2}$. More precisely, suppose that $L$ is such that $\pi c L>20$ and $\pi c^{2} L<1$ (e.g choose $\left.L \sim c^{-3 / 2}\right)$. Then for any (arbitrarily smooth) initial data $\omega_{0}$ with $\omega_{0}(k, 0)=1 \geq 0.5 \max _{\eta}\left|\omega_{0}(k, \eta)\right|$ it holds that

$$
\omega\left(j-\frac{1}{2}, k, j\right) \geq d^{j}
$$

for $d=\frac{\pi}{10} c L>1$ and all $j \in \mathbb{N}$.

This shows that phase-mixing for smooth Bilipschitz profiles by itself is not strong enough to prevent an exponential instability. Some smallness or non-resonance is necessary. While the embedding eigenvalue criterion of [WZZ18] provides a very good description of this, we think the present method of proof of constructing a sustained echo chain provides an important new perspective on this instability.

Proof. By Lemma 5 the Duhamel iteration converges for all times and is controlled by an exponential series. In the following we use the more precise control of the Duhamel iterates as sums over all paths (see Definition 6 and Lemma 7) starting in a frequency $\eta_{0}$ and ending in a frequency $\eta_{1}$.

We recall that our equation (5)

$$
\partial_{\tau} \omega(\eta)+\frac{c}{2} \frac{1}{k^{2}+(\eta+1-\tau)^{2}} \omega(\eta+1)-\frac{c}{2} \frac{1}{k^{2}+(\eta-1-\tau)^{2}} \omega(\eta-1)=0,
$$

considers only nearest neighbor interactions. 
In the following we argue by an iteration scheme, where we consider the sequence of times $T_{j}=j-\frac{1}{2}, j \in \mathbb{N}$. We claim that if

$$
\left|\omega\left(T_{j}, k, j\right)\right| \geq 0.5 \max _{\eta}\left|\omega\left(T_{j}, k, \eta\right)\right|
$$

then it holds that

$$
\left|\omega\left(T_{j+1}, k, j+1\right)\right| \geq 0.5 \max _{\eta}\left|\omega\left(T_{j+1}, k, \eta\right)\right|,
$$

and additionally

$$
\left|\omega\left(T_{j+1}, k, j+1\right)\right| \geq d\left|\omega\left(T_{j}, k, j\right)\right| .
$$

The result then follows immediately by induction in $j$.

Let thus $j \in \mathbb{N}$ be given and suppose that (14) holds. The proof of (15) and (16) then consist of establishing an upper bound on $\omega\left(T_{j+1}, k, \eta\right)$ for all frequencies $\eta$ (to control the maximum) as well as a lower bound for $\eta=j+1$. We will show that $\omega\left(T_{j+1}, k, \eta\right)$ satisfies the following convolution inequality

$$
\begin{aligned}
\left|\omega\left(T_{j+1}, \eta\right)-\omega\left(T_{j}, \eta\right)\right| \leq & \frac{1}{1-2 \delta}(2 \delta)^{\operatorname{dist}(\cdot, \cdot)} *\left|\omega\left(T_{j}, \cdot\right)\right| \\
& +C r(2 \delta)^{|\eta-j \pm 1|} \sum_{\eta_{0}}(2 \delta)^{\left|\eta_{0}-j\right|}\left|\omega\left(T_{j}, \eta_{0}\right)\right|,
\end{aligned}
$$

where $r \approx c \pi L>1$ is a large constant (see (22) for the definition), $C=\frac{1}{(1-2 \delta)^{3}} \frac{1}{1-2 r \delta}$ is close to 1 and $|\eta-j \pm 1|:=\min (|\eta-j+1|,|\eta-j-1|)$. We note that here $\eta_{0}=j$ and $\eta \in\{j-1, j+1\}$ have a distinguished role and will also prove that

$$
\left|\omega\left(T_{j+1}, j+1\right)-r \omega\left(T_{j}, j\right)\right| \leq \frac{1}{4} r \omega\left(T_{j}, j\right)
$$

and hence

$$
\left|\omega\left(T_{j+1}, j+1\right)\right| \geq \frac{3}{4} r\left|\omega\left(T_{j}, j\right)\right| .
$$

Supposing for the moment that (17) holds, we may take the supremum in $\eta$ and further estimate

$$
\begin{gathered}
\sup _{\eta}(2 \delta)^{\operatorname{dist}(\cdot, \cdot)} *\left|\omega\left(T_{j}, \cdot\right)\right| \leq \frac{1}{1-2 \delta} \sup _{\eta}\left|\omega\left(T_{j}, \eta\right)\right|, \\
\sum_{\eta_{0}}(2 \delta)^{\left|\eta_{0}-j\right|}\left|\omega\left(T_{j}, \eta_{0}\right)\right| \leq \frac{1}{1-2 \delta} \sup _{\eta_{0}}\left|\omega\left(T_{j}, \eta_{0}\right)\right|,
\end{gathered}
$$

and therefore by (14)

$$
\sup \left|\omega\left(T_{j+1}, k, \eta\right)\right| \leq\left(1+\frac{1}{1-2 \delta}+C r \frac{1}{1-2 \delta}(2 \delta)^{|\eta-j \pm 1|}\right) 2\left|\omega\left(T_{j}, k, j\right)\right| .
$$

For $r$ sufficiently large the last term in this upper bound is of greatest interest. It is comparable to comparable to $r\left|\omega\left(T_{j}, k, j\right)\right|$ if $\eta \in\{j-1, j+1\}$, which up to a factor matches our lower bound (19). In order to establish the estimates (15) and (16) we now additionally note that for $\eta \notin\{j-1, j+1\}$ the exponential $(2 \delta)^{|\eta-j \pm 1|}$ is small and can counteract the loss of a factor $2 / \frac{3}{4}$. 
It thus remains to prove (17) and (19). We again begin by studying the case when $\omega_{0}\left(T_{j}\right)$ be given by a single mode:

$$
\omega\left(t_{j}, k, \eta\right)=\delta_{\eta_{0}}(\eta)
$$

Then using Lemma 7 for any $\eta$ we may compute

$$
\omega\left(T_{j+1}, k, \eta\right)-\omega\left(T_{j}, k, \eta\right)=\sum_{\gamma: \gamma_{0}=\eta_{0}, \gamma_{|\gamma|}=\eta} I_{\gamma}\left[T_{j}, T_{j+1}\right] .
$$

While in the proof of Theorem 8 we roughly estimated

$$
I_{\gamma}\left[T_{j}, T_{j+1}\right] \leq d^{|\gamma|},
$$

in the following we estimate more carefully. In each integral we have to consider terms of the form

$$
\int_{\tau_{i-1} \leq \tau_{i} \leq \tau_{i+1}} \frac{c}{k^{2}+\left(\gamma_{i}-\tau_{i}\right)^{2}} d \tau_{i} \leq \int_{T_{j} \leq \tau_{i} \leq T_{j+1}} \frac{c}{k^{2}+\left(\gamma_{i}-\tau_{i}\right)^{2}} d \tau_{i} .
$$

If $\left|\gamma_{i}-j\right| \geq 1$, we can bound this contribution by

$$
\int_{-\frac{1}{2}}^{\frac{1}{2}} \frac{c}{k^{2}+\frac{1}{4}} d \tau_{i} \leq 4 c=: \delta<\frac{1}{4} .
$$

We call such $\gamma_{i}$ non-resonant. We recall that by Remark 2 that we only need to consider $\gamma_{i} \in \mathbb{Z}$ and thus there is a clean dichotomy: Either $\gamma_{i} \neq j$ is non-resonant or $\gamma_{i}=j$ is (perfectly) resonant.

In the following we thus group paths by the number of resonances appearing. Similarly to the proof of Theorem 8 , if all $\gamma_{i}$ in a path $\gamma$ are non-resonant, we may estimate

$$
I_{\gamma}\left[T_{j}, T_{j+1}\right] \leq \delta^{|\gamma|}
$$

Again roughly estimating the number of all paths of length $|\gamma|$ by $2^{|\gamma|}$, the sum over all such paths can be estimated by a geometric series:

$$
\sum_{\gamma: \text { non-resonant }} I_{\gamma}\left[T_{j}, T_{j+1}\right] \leq \sum_{i \geq \operatorname{dist}\left(\eta, \eta_{0}\right)} 2^{i} \delta^{i}=\frac{1}{1-2 \delta}(2 \delta)^{\operatorname{dist}\left(\eta, \eta_{0}\right)} .
$$

We remark that here the distance refers to the shortest non-trivial path, thus $\operatorname{dist}\left(\eta, \eta_{0}\right)=\left|\eta-\eta_{0}\right|$ if $\eta \neq \eta_{0}$ and $\operatorname{dist}\left(\eta_{0}, \eta_{0}\right)=2$. As in the proof of Theorem 8 in the case of general initial data (that is, not just a single mode) this estimate results in a discrete convolution against $\left|\omega\left(T_{0}, k, \cdot\right)\right|$, which produces the first term in the estimate (17).

In contrast, consider the special case $\eta_{0}=j$ and $\eta=j+1$. Then the path $\gamma=\left(\eta_{0}, \eta_{0}+1\right)$ has the associated integral given by

$$
\int_{-\frac{1}{2}}^{\frac{1}{2}} \frac{c}{k^{2}+\tau^{2}} d \tau=\frac{2 c}{k} \arctan \left(\frac{1}{2 k}\right)=: r .
$$

If $k<c$ is sufficiently small, then $2 \arctan \left(\frac{1}{2 k}\right) \approx \pi$ and $r \approx c \frac{\pi}{k} \geq c \pi L>1$ is comparatively large.

More generally, let $\gamma$ be a path starting in $\eta_{0}$ and ending in $\eta$. 
- If all $\gamma_{i}$ are non-resonant, this path is estimated by (21). Thus suppose that it has several resonances.

- We note that in order to be resonant, the path first has to reach $j$ starting from $\eta_{0}$, for which it needs $j_{1} \geq\left|\eta_{0}-j\right|$ steps.

- It is then resonant for a number $j_{2} \geq 1$ of times, between which is nonresonant a number at least $j_{3} \geq j_{2}-1 \geq 0$ times, since subsequent entries in a path are distinct, that is you have to leave the resonant frequency before visiting again.

- Finally, after visiting the resonant frequency a last time, the path has to reach the frequency $\eta$ for which it uses $j_{4} \geq|\eta-j \pm 1|$ non-resonant steps (for example the path $\gamma=(j, j-1)$ has a single resonance and no non-resonance).

Thus, in total the integral corresponding to this path can be estimated by

$$
\begin{aligned}
\delta^{j_{1}} r^{j_{2}} \delta^{j_{3}} \delta^{j_{4}} & =\delta^{j_{1}+j_{4}} r^{1}(r \delta)^{j_{2}-1} \delta^{j_{3}-j_{2}+1}, \\
j_{1} \geq\left|\eta_{0}-j\right|, \quad j_{4} & \geq|\eta-j \pm 1|, \\
j_{2}-1 \geq 0, \quad j_{3}-j_{2}+1 & \geq 0,
\end{aligned}
$$

where we use the short notation $|\eta-j \pm 1|=\min (|\eta-j+1|,|\eta-j-1|)$. We note that by assumption

$$
\begin{aligned}
r \delta \leq 8 \pi c^{2} L & <\frac{1}{4}, \\
\delta & <\frac{1}{4}, \\
r & >10 .
\end{aligned}
$$

Again roughly estimating the number of such paths by $2^{|\gamma|}$, we estimate the sums in $j_{2}-1$ and in $j_{3}-j_{2}+1$ by

$$
\frac{1}{1-2 r \delta} \frac{1}{1-2 \delta} \leq 4
$$

and similarly estimate the sums in $j_{1}, j_{4}$ by

$$
\frac{1}{(1-2 \delta)^{2}}(2 \delta)^{\left|\eta_{0}-j\right|+|\eta-j \pm 1|}
$$

We thus in total obtain an estimate by

$$
\frac{1}{(1-2 \delta)^{3}} \frac{1}{1-2 r \delta} r(2 \delta)^{\left|\eta_{0}-j\right|+|\eta-j \pm 1|} .
$$

Denoting $C=\frac{1}{(1-2 \delta)^{3}} \frac{1}{1-2 r \delta}$, we may again argue as in the proof of Theorem 8 to obtain an estimate for the case of general initial data in terms of a convolution in $\eta_{0}$. This concludes the proof of the estimate (17).

It remains to prove (19). We saw in (21) that the contribution by the path $\gamma=(j, j+1)$ (and analogously $(j, j-1)$ ) is given by

$$
r \omega\left(T_{j}, j\right) \text {. }
$$

In constrast all other paths ending in $j+1$ involve at least one non-resonance (and more the further $\eta$ or $\eta_{0}$ are from $j$ ). Hence, we further obtain the following lower 
bound:

$$
\begin{aligned}
& \left|\omega\left(T_{j+1}, j+1\right)-\omega\left(T_{j}, j+1\right)-r \omega\left(T_{j}, j\right)\right| \\
\leq & \frac{1}{1-2 \delta} \sup _{\eta}\left|\omega\left(T_{j}\right)\right|+C r \sum_{\eta_{0} \neq j}(2 \delta)^{\eta_{0}-j}\left|\omega_{0}\left(T_{j}, \eta_{0}\right)\right| \\
\leq & \frac{1}{1-2 \delta} \sup _{\eta}\left|\omega\left(T_{j}\right)\right|+C r(2 \delta) \frac{1}{1-2 \delta} \sup _{\eta_{0}}\left|\omega\left(T_{j}, \eta_{0}\right)\right|,
\end{aligned}
$$

where we used the geometric series in the last step. Since $\omega\left(T_{j}, j\right)$ is comparable to the supremum at that time by the assumption (14) and using that $r \gg 1$ and choosing $\delta$ sufficiently small such that $C(2 \delta) \frac{1}{1-2 \delta} \ll 1$, we deduce that

$$
\left|\omega\left(T_{j+1}, j+1\right)-\omega\left(T_{j}, j+1\right)-r \omega\left(T_{j}, j\right)\right| \leq 0.1 r\left|\omega\left(T_{j}, j\right)\right|
$$

which implies (19).

In this theorem we have shown that if a torus is sufficiently long such that $c L \gg 1$, then a new resonance cascade mechanism persists, where $(k, \eta=j)$ excites a mode $(k, j+1)$ around time $T_{j}$, which in turn excites $(k, j+2)$ and so on. We thus present a new explicit instability mechanism associated to the smallness assumption imposed in [Zi116]. Furthermore, similarly to convexity/concavity assumptions imposed in classical stability results by Rayleigh, Fjortoft or Arnold [Dra02], this result shows that even if a flow is bilipschitz and smooth, this is not sufficient to establish (asymptotic) stability and that some additional condition to control long wavelength perturbations is necessary. We thus develop a further understanding of what (linear) instability mechanisms may be encountered in the study of perturbations to shear flows, how instabilities may propagate and cascade and what conditions may be imposed to avoid these scenarios (e.g. limiting the wave length of admissible perturbations). Here an interesting but very challenging question concerns the implications of this linear instability mechanism for the nonlinear problem. While such an instability rules out some types of asymptotic convergence results (that is, scattering to the linear dynamics), it might be the case that the linearization around the initial velocity profile is the "wrong guess" for the asymptotics and that the evolution is nevertheless asymptotically stable.

In view of the nonlinear problem we remark that the $\eta$-chain model of the present article and the fluid echo chains studied in [DM18] and [DZ19] share commonalities in how instabilities appear at critical times and then lead to new instabilities at later times, resulting in a cascade. However, as remarked earlier the details such as the direction (in Fourier space), length or time scales of these cascades are very different. Furthermore, in the general nonlinear problem one may expect both mechanisms to interact, resulting in resonances in further directions.

\section{REFERENCES}

[Arn66] Vladimir Igorevich Arnold. Sur la géométrie différentielle des groupes de Lie de dimension infinie et ses applications à l'hydrodynamique des fluides parfaits. Ann. Inst. Fourier, 16(1):319-361, 1966.

[BCV17] J. Bedrossian, M. Coti Zelati, and V. Vicol. Vortex axisymmetrization, inviscid damping, and vorticity depletion in the linearized 2D Euler equations. ArXiv e-prints, November 2017.

[BM15] Jacob Bedrossian and Nader Masmoudi. Inviscid damping and the asymptotic stability of planar shear flows in the 2D Euler equations. Publ. Math. Inst. Hautes Études Sci., 122:195-300, 2015. 
[BMM16] Jacob Bedrossian, Nader Masmoudi, and Clément Mouhot. Landau damping: paraproducts and Gevrey regularity. Annals of PDE, 2(1):4, 2016.

[CZZ19] Michele Coti Zelati and Christian Zillinger. On degenerate circular and shear flows: the point vortex and power law circular flows. Communications in Partial Differential Equations, 44(2):110-155, 2019.

[DM18] Yu Deng and Nader Masmoudi. Long time instability of the Couette flow in low Gevrey spaces. arXiv preprint arXiv:1803.01246, 2018.

[Dra02] Philip G. Drazin. Introduction to Hydrodynamic Stability. Cambridge University Press, 2002.

[DZ19] Yu Deng and Christian Zillinger. Echo chains as a linear mechanism: Norm inflation, modified exponents and asymptotics. arXiv preprint arXiv:1910.12914, 2019.

[FH98] Susan Friedlander and Louis Howard. Instability in parallel flows revisited. Studies in Applied Mathematics, 101(1):1-21, 1998.

[Jia19] Hao Jia. Linear inviscid damping in Gevrey spaces. arXiv preprint arXiv:1904.01188, 2019.

[Lin03] Zhiwu Lin. Instability of some ideal plane flows. SIAM journal on mathematical analysis, 35(2):318-356, 2003.

[LZ11] Zhiwu Lin and Chongchun Zeng. Inviscid dynamical structures near Couette Flow. Archive for rational mechanics and analysis, 200(3):1075-1097, 2011.

[MS61] LD Meshalkin and Ia G Sinai. Investigation of the stability of a stationary solution of a system of equations for the plane movement of an incompressible viscous liquid. Journal of Applied Mathematics and Mechanics, 25(6):1700-1705, 1961.

[MV11] Clément Mouhot and Cédric Villani. On Landau damping. Acta mathematica, 207(1):29201, 2011.

[Orr07] William M'F Orr. The stability or instability of the steady motions of a perfect liquid and of a viscous liquid. In Proceedings of the Royal Irish Academy. Section A: Mathematical and Physical Sciences, pages 69-138. JSTOR, 1907.

[WZZ17] D. Wei, Z. Zhang, and W. Zhao. Linear inviscid damping and enhanced dissipation for the Kolmogorov flow. ArXiv e-prints, November 2017.

[WZZ18] Dongyi Wei, Zhifei Zhang, and Weiren Zhao. Linear inviscid damping for a class of monotone shear flow in Sobolev spaces. Communications on Pure and Applied Mathematics, 71(4):617-687, 2018.

[Zil16] Christian Zillinger. Linear inviscid damping for monotone shear flows in a finite periodic channel, boundary effects, blow-up and critical Sobolev regularity. Arch. Ration. Mech. Anal., 221(3):1449-1509, 2016.

[Zil17] Christian Zillinger. On circular flows: linear stability and damping. J. Differential Equations, 263(11):7856-7899, 2017.

USC Dornsife, Department of Mathematics, 3629 S. Vermont Ave., CA 90089 Los Angeles, USA

Email address: yudeng@usc.edu

BCAM - Basque Center for Applied Mathematics, Mazarredo, 14 E48009 Bilbao, BASque Country - SPAIN

Email address: czillinger@bcamath.org 